\title{
LAS SALIDAS DE CAMPO EN ESPAÑA COMO RECURSO DIDÁCTICO PARA LA ENSEÑANZA DE LA GEOGRAFÍA. UNA REVISIÓN BIBLIOGRÁFICA
}

\author{
Álvaro Francisco Morote Seguido \\ Departamento de Didáctica de las Ciencias Experimentales y Sociales \\ Facultad de Magisterio \\ Avda. Tarongers 4, 46022 Valencia (España) \\ Universidad de Valencia \\ alvaro.morote@uv.es
}

\begin{abstract}
Resumen: La enseñanza de la Geografía debe orientarse hacia la formación de estudiantes que posean las destrezas de comprender y analizar el territorio a través de la localización y distribución de hechos relevantes, así como que sean capaces de obtener, analizar y aplicar dicha información a los diferentes contextos de la vida cotidiana. El objetivo de este trabajo es realizar una revisión bibliográfica de trabajos de investigación sobre las salidas de campo en España desde el campo de la Geografía e identificación de las principales temáticas de estudio (1980-2018). Tras el análisis de los resultados, se han identificado 4 temáticas principales: 1) La potencialidad que tienen las salidas de campo como recurso didáctico para la enseñanza de la Geografía; 2) La importancia de estos recursos para la interpretación y análisis del territorio; 3) Trabajos de propuestas de salidas de campo (estudios de caso); y 4) La incorporación de las Tecnologías de la Información y la Comunicación a estos recursos. Con esta revisión bibliográfica se ha podido comprobar la evolución de las diferentes temáticas de estudio en las últimas décadas y valorar como las salidas de campo pueden ayudar al alumnado a asimilar conceptos teóricos, crear una visión crítica e interpretativa del territorio analizado.
\end{abstract}

Palabras clave: Salidas de campo, recurso didáctico, enseñanza, Geografía.

Abstract: The teaching of Geography should be directed towards about the formation of students who possess the skills to understand and analyze the territory through the location and distribution of relevant facts, as well as

Recibido: 21-09-2018. Aceptado: 05-05-2019. 
being able to obtain, interpret, value and apply such information to the different contexts of everyday life. The aim of this research is to do a bibliometric review and analyze the evolution of studies about fieldworks in Spain from the Geography and the identification of the main topics of research (1980-2018). The results reveal 4 main themes: 1) The potential of fieldwork as a didactic resource for the teaching of Geography; 2) The importance of fieldworks for the interpretation of the territory; 3) Proposals for fieldworks (case studies); and 4) The incorporation of the Information and Communication Technologies to fieldworks. After this bibliographical review, it becomes clear how the fieldworks can help the student to assimilate theoretical concepts, create a critical and interpretative vision of the territory analyzed, and review the evolution of these topics in the last decades.

Keywords: Fieldworks, instructional resource, learning, Geography.

\section{Introducción}

La Geografía aporta en la educación la dimensión espacial del conocimiento, facilitando la comprensión de la información, conceptos, procedimientos y actitudes referentes a la localización y distribución de diferentes hechos sobre el espacio (Crespo, 2012; Souto, 2010). La Geografía es la ciencia de las relaciones entre el hombre (la sociedad) y la naturaleza (el medio). Su concreción se manifiesta siempre en un espacio concreto (un territorio) cuya apariencia fisionómica da lugar a un paisaje que, a su vez, es una elaboración cultural (Martínez-Fernández et al., 2015). Además, esta ciencia permite la adquisición de habilidades para el análisis de la ocupación del territorio por el ser humano y valorar críticamente el espacio resultante de esa transformación. Por lo tanto, la enseñanza de la ciencia geográfica debe orientarse hacia la formación de estudiantes que posean las destrezas de comprender y analizar el territorio a través de la localización y distribución de hechos relevantes, así como que sean capaces de obtener, interpretar, valorar y aplicar dicha información a los diferentes contextos de la vida cotidiana (Sebastià y Tonda, 2011). Como indican Martínez-Fernández et al. (2015: 137) "con mayor o menor acierto, la Geografía escolar no solo se limita ya a un juego de localizaciones espaciales sobre un mapa, como se hacía prácticamente en exclusiva en el pasado, sino que plantea la reflexión geográfica en aras a dar una explicación ordenada de las diferentes configuraciones espaciales de la superficie terrestre a escala mundial, regional o locat'.

Diversos estudios indican que el uso del libro de texto y de la metodología expositiva clásica siguen siendo predominantes en las clases de Ciencias Sociales (Martínez et al., 2009) y, especialmente en las de Geografía (De Miguel, 2013b), con los efectos que ello tiene de convertirse en una asignatura ardua, extensa, compleja y poco atractiva para el alumnado (Morote, 2017). Por ello, una forma de romper con las rutinas escolares es el 
uso de salidas de campo debido a su diversidad, apoyo en recursos visuales y dinámicos que hacen de esta ciencia una materia más atractiva en la que el alumnado se encuentra más motivado para el aprendizaje (Sandoya, 2005; Boyle et al., 2007). Diferentes autores (Piñeiro, 1997; Morote y Moltó, 2017) han puesto de manifiesto y propuesto la importancia de la realización de las salidas de campo, entendidas éstas como cualquier actividad realizada por el profesor y los alumnos sobre el terreno. Aunque, dentro de esta definición tan general se encuentran múltiples formas que, tradicionalmente han recibido nombres distintos como trabajos de campo, jornadas de campo, excursiones, etc. Los estudios sobre las salidas de campo se trata de una temática con una dilatada experiencia a escala internacional como ponen de manifiesto los trabajos llevados a cabo en Europa (Anđelkovi et al., 2018; Grubar et al., 2018; Marvell y Simm, 2018) y, especialmente, en Reino Unido (France y Haigh, 2018; Tilling, 2018; Tucker y Horton, 2019).

Como explican Ortega (1998) y Crespo (2012), a finales del siglo XIX, en España, fue la Institución Libre de Enseñanza la que adoptó las excursiones como parte fundamental de su nuevo enfoque pedagógico, ya que en ella se daban las mejores condiciones para lograr la educación completa e integradora del ser humano. En 1886, Francisco Giner de los Ríos y Manuel Bartolomé Cossío, realizaron la primera excursión pedagógica de la Institución a la sierra madrileña, hecho que supondría una nueva puesta en valor del paisaje serrano, el denominado "guadarramismo". Sin embargo, como indica Souto (2010) aún, la Geografía tradicional que se transmite a través de las prácticas escolares y la propia opinión pública se resiste a las propuestas de innovación que pretenden responder a las exigencias del contexto socio-ambiental para superar los nuevos desafíos ciudadanos. Como resultado, algunos conceptos que se aprenden en el aula como el espacio geográfico, espacio o parque natural se enfrentan con la información que el alumnado recibe constantemente desde su propia realidad vivida (Santana et al., 2015). Ello reafirma lo que muchos autores siguen subrayando: La conveniencia de las salidas de campo para confrontar la realidad con las ideas mentales del alumnado (Morote y Moltó, 2017). Con ello se superarían las tradicionales "salidas al entorno natural" para pasar a acciones concretas basada en itinerarios didácticos interdisciplinares con una finalidad transformadora (García de la Vega, 2004; Vilarrasa, 1999; 2003a). Hasta hace poco tiempo, las salidas de campo se veían como una actividad no escolar, sin embargo, el logro de mayores niveles de renta durante las últimas décadas ha permitido que, en la actualidad, los ciudadanos que se integran en la enseñanza reglada y las administraciones públicas, consideren con clara coincidencia y fines educativos la progresiva inversión en recursos proyectados en actividades extraescolares, programas educativos, intercambios y experiencias, exposiciones, etc. (Morote, 2017).

Como afirman Moreno y Cely (2013), las salidas de campo pueden tener múltiples intencionalidades que justifican procesos construidos en el inicio, transcurso y al final de un escenario escolar específico ya que permite ampliar el horizonte de habilidades y conocimientos propios en el desarrollo del aprendizaje. Según Delors (1997) las salidas de campo como estrategia de enseñanza y de aprendizaje facilita el cumplimiento 
de los cuatro pilares de la educación propuestos por la UNESCO en cuanto a: 1) Aprender a ser; 2) Aprender a convivir; 3) Aprender a conocer; y 4) Aprender a hacer. Respecto a la experiencia del aprendizaje, ésta posibilita la apropiación y comprensión de conceptos y lenguajes por medio de la vivencia del lugar y por otro, permite contrastar estos conocimientos con la realidad, con lo observable, con la percepción y apropiación de los conocimientos mediante la experiencia que enriquecerá la formación y la práctica pedagógica. También cabe indicar que las salidas de campo ofrecen un laboratorio del territorio donde el alumnado puede aprender, de forma crítica, el funcionamiento y problemas de la sociedad actual. Por ello, gracias a este recurso, el alumnado se ve capacitado para analizar los problemas sociales y ambientales más importantes, para llegar a comprender la dinámica de las sociedades y, para ser capaces como futuros ciudadanos, de afrontar de manera crítica y comprometida los problemas fundamentales que se suceden. También cabe indicar que una novedad en las salidas de campo es el papel en las iniciativas de investigación. En este sentido, como argumentan Álvarez et al. (2016), las salidas de campo son una oportunidad para que los estudiantes desarrollen habilidades como la observación, la problematización y construcción de conocimientos socio-espaciales que pueden afectar su vida cotidiana. En definitiva, un espacio de reflexión y aprendizaje sobre la realidad.

Zusman (2011) explica que la Geografía ha tenido cuatro formas de concebir las salidas de campo y que cada una de ellas supone una manera particular de entender esta ciencia. La primera de ellas está asociada a las actividades de exploración desarrolladas a finales del siglo XIX y principios del XX en el marco de los procesos de apropiación territorial. Bajo el supuesto de que la Geografía de la primera mitad del siglo XX estudiaba las regiones o los paisajes, la segunda concepción vincula las salidas de campo a la necesidad de definir una metodología propia diferenciada de la de otros saberes. La tercera propuesta, en la segunda mitad del siglo XX (años setenta y ochenta), se construye desde la postura que sostiene que la Geografía es una disciplina social comprometida con la transformación social. Ello, según este autor, derivó en una visión de trabajo de campo que suponía la necesidad de involucrarse con las problemáticas sociales cotidianas. Y, finalmente, la introducción del método etnográfico en la Geografía (década de los noventa), que ha llevado a redefinir las relaciones entre la academia y las salidas de campo y a promover la reflexión del investigador sobre las implicaciones políticas y sociales de estos recursos didácticos. A estas 4 formas de concebir las salidas de campo, en la última década se asiste a una transformación de dicho recurso con el uso de nuevas tecnologías como el Global Positioning System (GPS) (geoposicionamiento), aplicaciones móviles compatibles con los Sistemas de Información Geográfica (IGN-Iberpix, Collector for ArcGIS, QField, etc.), que hacen más motivadora, si cabe, este tipo de recursos fuera del aula (García y Delgado, 2013; Fuller et al., 2006; Fombona y Pascual, 2016; Moreno et al., 2015).

Sin embargo, a pesar de que las herramientas cartográficas han cambiado notablemente con la aparición de internet (SIG, Google maps, GPS, Iberpix, etc.), ese cambio, actual- 
mente no es del todo perceptible en las clases de Geografía, ya que los profesores apenas recurren a estas nuevas herramientas de cartografía móvil, con mapas que se descargan en el smartphone, tablet o navegador de los alumnos (Moreno y Vera, 2017). Como ponen de manifiesto estos autores, la aparición del Web-mapping está, lógicamente, unida a la extensión de uso de internet, la Web 2.0 y, en el último lustro, a las aplicaciones móviles que se instalan en los smartphones. Por ejemplo, en Reino Unido y los EE.UU., a mediados de 2005 los principales sitios de Web-mapping tenían una cifra de visitantes de 47 millones, mientras que en 2007, esa cifra había ascendido a los 71 millones de usuarios de Google Maps y los 22 millones que ya usaban Google Earth (Haklar et al., 2008).

Actualmente, los trabajos sobre experiencias didácticas que utilizan los códigos QR en los dispositivos móviles son muy escasos ya que se trata de una tecnología muy reciente y poco extendida en las aulas, además de que hay que sumar el inconveniente de la prohibición de los teléfonos móviles en clase (Moreno y Vera, 2017). Respecto al uso de esta herramienta en las salidas de campo, por ejemplo, fuera de España destacan los trabajos de Rikala y Kankaaranta (2013) y de Rikala (2015), en el que se pone de manifiesto el potencial didáctico para el aprendizaje que tienen estos códigos, a pesar de que aún el profesorado es reticente a utilizarlos. Según Rikala y Kankaraanta (2013) estos códigos permiten despertar la curiosidad del alumnado y variar los métodos de enseñanza centrándose más en los estudiantes. En relación con el uso de las TIC en clase, de manera general, para lograr una mayor implantación de su uso (no sólo en las salidas de campo sino también dentro del aula), Gómez-Trigueros (2010) explica que el nuevo modelo de enseñanza tiene que basarse en tres pilares fundamentales: 1) Investigar y descubrir la información; 2) Emplear como medio de expresión las nuevas tecnologías; y 3) Compartir y comunicar sus conocimientos a través de la red.

Respecto al objetivo de este trabajo, éste es analizar la evolución de la producción científica sobre las salidas de campo en España desde el campo de la Geografía e identificar las principales líneas de estudio y autores (1980-2018) y comprobar aquellas que ganan o pierden importancia.

\section{Metodología}

Para llevar a cabo la consecución del objetivo propuesto se ha procedido a una revisión bibliográfica de estudios que han tratado la potencialidad de las salidas de campo en España como recurso didáctico para la enseñanza de la Geografía. El primer paso metodológico ha consistido en la definición de diferentes palabras clave que se insertaban tanto en el título, palabras clave y en el resumen de las investigaciones (tanto en castellano e inglés): Salida de campo; jornada de campo; trabajo de campo; Tecnologías de la Información y la Comunicación; Geografía; España. Las bases de datos consultadas han sido Dialnet, Scielo, Scopus y Web of Science. 
Para acotar el número de trabajos cabe indicar que para este trabajo se han tenido en cuenta los artículos, libros y capítulos de libro que trataban las salidas de campo en España. Cabe poner de manifiesto que no se han tenido en cuenta las actas de congresos debido a que un gran número de ellas no se adscriben en las bases de datos, principalmente las del pasado s. XX. De ahí la complejidad a la hora de poder consultar todas las obras producidas en congresos o reuniones científicas que tuvieran dentro de sus descriptores las palabras clave utilizadas. El marco temporal elegido ha sido 19802018. La elección de esta fecha se justifica para poder comprobar la importancia e interés mostrado desde esa fecha por los autores que han tratado esta temática debido al fomento de la Didáctica de la Geografía en los años ochenta. Al respecto, en España, la Didáctica de la Geografía es una disciplina relativamente reciente, debido a su adscripción al área de conocimiento de la Didáctica de las Ciencias Sociales, tras su creación por el Real Decreto de finales de 1984, en desarrollo de la Ley de reforma universitaria y en la que tuvo un gran impulso gracias a la creación del Grupo de Didáctica de la Geografía de la Asociación de Geógrafos Españoles en 1986 (De Miguel, 2017), recientemente llamada como Asociación Española de Geografía.

En la primera búsqueda bibliográfica, el número total de trabajos identificados fue de 130. A continuación, se procedió a una depuración de estas investigaciones teniendo en cuenta los criterios establecidos: 1) Trabajos de Geografía en relación con las salidas de campo; 2) Investigaciones como marco geográfico el territorio español; y 3) Trabajos que se adscriben a artículos, libros y capítulos de libro. Finalmente, tras esta segunda criba, el número total ascendió a 90. Los resultados se exponen siguiendo el modelo de otros trabajos como el de Gómez-Ruiz (2010) en el que se incide en la vinculación entre salidas de campo y procedimientos para el aprendizaje escolar de la Geografía. Finalmente, cabe destacar que, en la descripción estadística de los resultados, se han tenido en cuenta todos los trabajos identificados, pero, debido al gran número de estudios, en el texto sólo se han citado y referenciado aquellos autores y estudios más influyentes en el campo de la Didáctica de la Geografía que han servido como referencia para trabajos posteriores.

\section{Resultados}

Los resultados obtenidos tras la revisión bibliográfica ponen de manifiesto que, desde los años ochenta del pasado siglo XX destacan 4 temas de estudio: 1) La potencialidad que tienen las salidas de campo como recurso didáctico para la enseñanza de la Geografía; 2) La importancia de las salidas de campo para la interpretación y análisis del territorio; 3) Trabajos de propuestas de salidas de campo (estudios de caso); y 4) La incorporación de las Tecnologías de la Información y la Comunicación a estos recursos. El análisis de la evolución temporal de la publicación de estos trabajos indica que ha habido una notable progresión desde la década de los ochenta y noventa pero a un diferente ritmo y evolución según temáticas. 
Respecto a la primera temática cabe indicar que se trata de una línea en la que se explica de manera general la potencialidad que tienen las salidas de campo para la ciencia geográfica. El número de trabajos asciende a 38 (la mayoría con el 42,22\%) (Figura 1). A partir de los noventa se convierte en una de las líneas de estudio con una publicación constante de trabajos. Algunos de éstos, como el de Gómez-Ortiz (1986) dio paso en la década siguiente a un aumento del interés por esta temática destacando los estudios de Melcón (1991), Martí (1994; 1996), García-Ruiz (1994; 1997), Friera (1995), Sánchez-Ogallar (1995), Fidalgo (1997) o Piñeiro (1997). Ya en el siglo XXI, durante la primera década destacan los trabajos llevados a cabo por Sánchez y Godoy (2002), Vilarrasa (2003a; 2003b), Ortega (2004), Umaña (2004), Pérez y Rodríguez (2006), Montilla (2005), Sandoya (2005) o Godoy y Sánchez (2007). En la última década aparecen otros trabajos como los de Souto (2010; 2011), Samper et al. (2013), García De la Vega (2014), Álvarez et al. (2016), Almuedo (2016), Sempere-Souvannavong et al. (2017) o García et al. (2018). El análisis de esta temática de estudio pone de manifiesto dos consideraciones: 1) Se trata de la principal temática en relación con las salidas de campo; 2) Su producción ha sido constante en las últimas cuatro décadas; y 3) Conforme han pasado los años, estos trabajos han perdido peso porcentual en detrimento de otras temáticas de estudio que han surgido (ver Figura 2). Además, también cabe explicar que un mismo autor o autores han trabajado diferentes temáticas como se podrá observar a continuación.

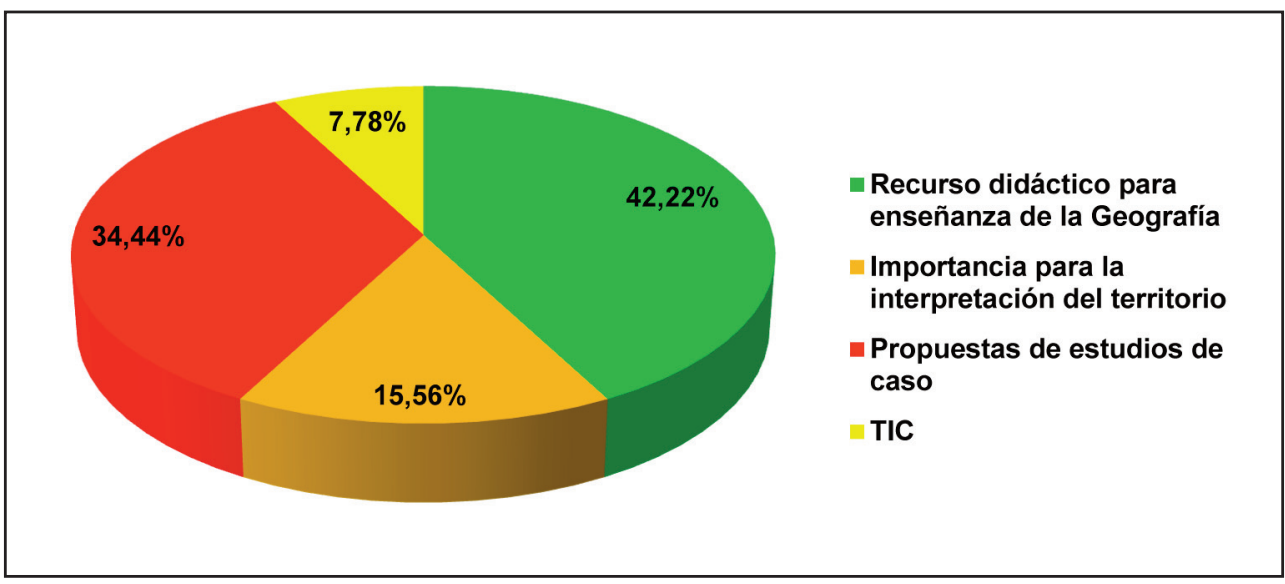

Figura 1. Trabajos identificados según temáticas de estudio (1980-2018).

Fuente: Elaboración propia.

De manera general, esta primera temática de estudio destaca por la potencialidad que tienen estos recursos para la enseñanza de la Geografía ya que permite poner en práctica y observar en la vida real lo visto en el aula. Desde la Declaración Internacional sobre Educación Geográfica (redactada en 1992 y actualizada en 2016) por la Comi- 
sion de Educacion Geografica de la Union Geografica Internacional se subrayó la necesidad de que el alumnado adquiriera conocimientos, habilidades y valores propios, es decir, contenidos conceptuales, procedimentales y actitudinales específicos de la educación geográfica (De Miguel y Fernández, 2016). Además, en dicha declaración se indicó que la educación geográfica estaba contribuyendo al desarrollo de la competencia social y ambiental, además de las intelectuales y personales, especialmente a través del dominio de habilidades y procedimientos propios del trabajo geográfico como las técnicas vinculadas al aprendizaje por descubrimiento (identificación de problemas espaciales, recogida, tratamiento e interpretación de información) y, como consecuencia, su expresión por medio de representaciones cartográficas (De Miguel, 2013a).

Las salidas de campo se presentan como una metodología de aprendizaje de lo más adecuada por las ventajas que ofrece. En la década de los ochenta, ya Gómez-Ortíz (1986: 109-116) argumentó las siguientes: 1) Integra el conocimiento del entorno en el currículo escolar; 2) Convierte al alumnado en el centro de la práctica y el aprendizaje; 3) Facilita la visión multidisciplinar del paisaje; 4) Consolida el trabajo en equipo del profesorado; 5) Fomenta en los estudiantes la observación directa de los objetos de estudio; 6) Colabora en la creación de un espíritu crítico, responsable y participativo en el alumnado; y 7) Permite captar la realidad de manera integrada. Una década más tarde, SánchezOgallar (1995: 160-184), sumó los siguientes valores: 1) Favorecen la conceptualización geográfica; 2) Permiten el desarrollo de destrezas procedimentales relacionadas con la medición o estimación de las distancias, inclinaciones, alturas, etc.; 3) Propician la comparación, poniendo de manifiesto semejanzas y diferencias entre distintas áreas; 4) Constituyen un marco único para el desarrollo de las destrezas cartográficas como la lectura de mapas, orientación o localización; y 5) Permiten a los discentes una perspectiva ambiental sobre el entorno que puede propiciar la búsqueda de soluciones a problemas ambientales. En la década de los noventa, por ejemplo, en el trabajo de Fidalgo (1997) se pone de manifiesto que las salidas de campo constituyen el medio más idóneo para que los estudiantes pongan a prueba la solidez de los conocimientos teóricos adquiridos, ejerciten las técnicas de observación, descripción, expresión gráfica y toma de datos y maduren su espíritu crítico en relación con los contenidos que le son transmitidos y con los resultados que ellos mismos pueden obtener de sus propios análisis.

Ya en el siglo XXI, según Godoy y Sánchez (2007), la realización de una salida de campo permite a los estudiantes lograr ciertas habilidades y destrezas: 1) Desarrollar la observación, el análisis y la síntesis; 2) Promover la autonomía del estudiante a la hora de desarrollar las actividades propuestas durante el trabajo (según el contenido dado); 3) Recoger información, directamente en el área de trabajo para la obtención de un producto científico; 4) Desarrollar habilidades y destrezas en el manejo de instrumentos y técnicas de muestreo; 5) Despertar inquietudes hacia el estudio de la disciplina y el desarrollo de la investigación; 6) Propiciar la formación de equipos interdisciplinares de trabajo; 7) Desarrollar en el estudiante la capacidad de presentar nuevas situaciones problemáticas en torno de un tópico; 8) Establecer buenas relaciones de trabajo entre los participantes; y 9) Aplicar los conocimientos adquiridos en el aula. Se- 
gún Montilla (2005) la salida de campo se trata de una herramienta didáctica valiosa para verificar en el terreno lo que teóricamente se expone en clase previamente. Por su parte, Hernández (2007) explica que las ventajas de estos recursos son incuestionables ya que permite: 1) Contacto directo con un determinado tipo de fuente; 2) Trabajo procedimental intenso; 3) Mejor adquisición de contenidos conceptuales y actitudinales; 4) Mayor motivación del alumnado; y 5) Enfrentamiento a problemas y casos reales. Lara (2011) argumenta que las salidas de campo se consideran una estrategia de enseñanza asociada a un aprendizaje grupal contextualizada en la realidad del espacio geográfico donde el profesor facilita la comprensión y el cambio conceptual en los discentes al despertar su interés y además, propicia el aprendizaje significativo.

Según Godoy y Sánchez (2007), la salida de campo para la enseñanza de la Geografía constituye una herramienta indispensable en cualquiera de sus ramas o ciencias auxiliares (geomorfología, climatología, meteorología, cartografía, antropología, entre otras), debido a que permite obtener experiencias significativas para comprender los fenómenos de la superficie terrestre en su vinculación con el espacio, sus diferenciaciones, cambios e interrelaciones causales. También, en palabras de estos autores, se tratan de una estrategia didáctica de enorme interés dentro del campo de la Geografía ya que al proyectar el aprendizaje en cualquiera de sus ámbitos desde el campo, el proceso se convierte "en algo emocionante tanto para el docente como para el estudiante y el campo es un laboratorio abierto que da la oportunidad de despertar en los alumnos inquietudes que les permiten descubrir un sin fin de información que aparece impresa en el paisaje" (Godoy y Sánchez (2007: 137). Además, permite vivenciar los fenómenos naturales a través del contacto directo con el ambiente donde se desenvuelven y ha sido considerado de vital importancia en la interpretación y análisis de los mismos desde la perspectiva científica (Sánchez y Godoy, 2002). Según Fidalgo (1997), su finalidad es doble: 1) Por una parte informativa, al descubrir en la complejidad de la naturaleza aquello que se ha visto simplificado en el aula; y 2) Formativa, al desplegar todas las capacidades del individuo para aprender la realidad.

En esta línea de estudio también cabe destacar el trabajo de Álvarez et al. (2016) en el que se explican las diferentes posibilidades de las salidas de campo teniendo en cuenta distintas dimensiones. Para el caso de la emocional, al trabajar con las percepciones e interacciones propias de los participantes, la formación también se puede generar desde un ámbito personal en el cual se involucran sentimientos, afectos y pensamientos que se transforman en relación directa con el escenario a explorar. En cuanto a la dimensión teórica, durante la salida de campo se puede visibilizar, por una parte, en el contraste de los debates teóricos con lo que se vive y por otra con la puesta en práctica de los enunciados presentados conceptualmente, para reafirmar o de construir lo teórico con lo vivido. En tercer lugar, la dimensión práctica, genera diferentes actividades que constituyen un escenario formativo, ya que es en la acción en la que se pueden identificar fortalezas y debilidades de los actores involucrados en la salida de campo. En acuerdo con la dimensión práctica, ésta conlleva a la vivencia real para poner en juego saberes, sentidos y significados de los participantes. Y, finalmente, la dimensión actitu- 
dinal, donde se pueden evidenciar en las actitudes y disposición que refleja cada uno de los actores involucrados en la salida que, genera enriquecimiento a partir de los conflictos cognitivos que surgen en el debate académico, epistemológico y convivencial permitiendo nuevos escenarios de interacción durante el trabajo de campo.

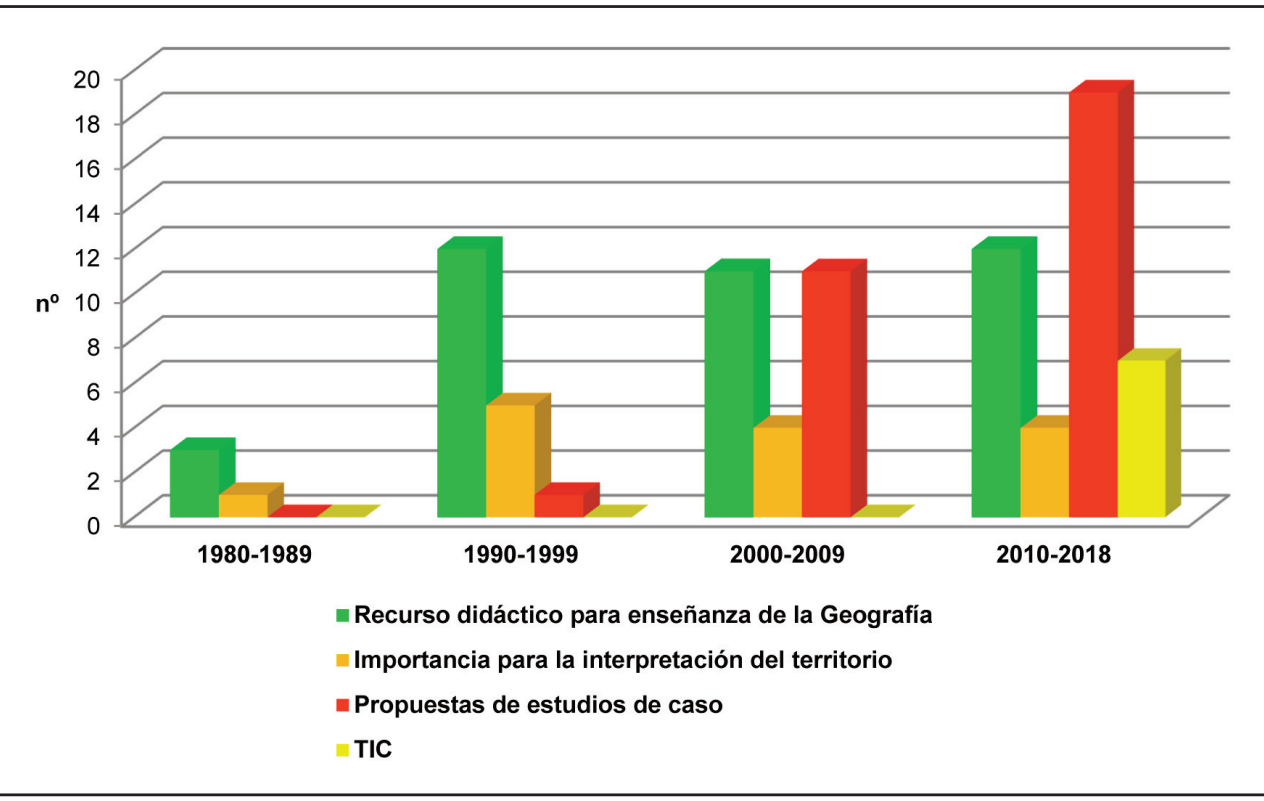

Figura 2. Evolución de los trabajos identificados según temáticas (1980-2018).

Fuente: Elaboración propia.

Una segunda línea identificada es la importancia que tienen las salidas de campo para la interpretación del territorio (análisis del paisaje, problemas ambientales, etc.). El número total de trabajos identificados asciende a 14 (el 15,56\%). Al igual que la primera temática, la producción de estos trabajos emerge en la década de los ochenta manteniéndose constante hasta la actualidad. Por ejemplo, en los ochenta destaca el trabajo de Meaza (1987), mientras que en los noventa aparecen Clary (1992), Villarrasa (1999), Souto (1998) o Bailey (1998). Éstos, se tratan de trabajos con el objetivo de conocer el medio, llevar a cabo un aprendizaje más cercano y vivido y aprender a situarse en el espacio. En el primer decenio del siglo XXI destacan los trabajos que se interesan por la potencialidad que tienen las salidas de campo para el estudio y valorización del paisaje como los de Ortega (2001), García De la Vega (2004), Parra et al. (2008), Sánchez-Ferrezuelo (2010), mientras que en el periodo 2010-2018 destacan por tratar conflictos territoriales y ambientales como los de García-Martín (2014), Santana et al. (2015), Cuello y Cuello (2016) o Ensabella (2016) (Cuadro 1). 
Cuadro 1. Evolución de trabajos según temática y autores (1980-2018)

\begin{tabular}{|c|c|c|c|c|}
\hline & $\begin{array}{c}\text { Potencialidad como } \\
\text { recurso didáctico }\end{array}$ & $\begin{array}{c}\text { Interpretación } \\
\text { del territorio }\end{array}$ & $\begin{array}{c}\text { Propuesta de } \\
\text { estudios de caso }\end{array}$ & TIC \\
\hline 1980-1989 & $\begin{array}{l}\text { Bailey (1981) } \\
\text { Morales (1984) } \\
\text { Gómez Ortiz (1986) }\end{array}$ & Meaza (1987) & & \\
\hline 1990-1999 & $\begin{array}{l}\text { Melcón (1991) } \\
\text { Wass (1992) } \\
\text { García Ruiz (1994) } \\
\text { Martí (1994) } \\
\text { Sánchez-Ogallar (1995) } \\
\text { Tatjer (1995) } \\
\text { Friera (1995) } \\
\text { Martí (1996) } \\
\text { Fidalgo (1997) } \\
\text { García-Ruiz (1997) } \\
\text { Piñeiro (1997) }\end{array}$ & $\begin{array}{l}\text { Clary (1992) } \\
\text { Liceras (1997) } \\
\text { Vilarrasa (1999) } \\
\text { Souto (1998) } \\
\text { Bailey (1998) }\end{array}$ & Palacios (1991) & \\
\hline 2000-2009 & $\begin{array}{l}\text { López (2000) } \\
\text { Sánchez y Godoy (2002) } \\
\text { Vilarrasa (2003a; 2003b) } \\
\text { Ortega (2004) } \\
\text { Umaña (2004) } \\
\text { Pérez y Rodríguez (2006) } \\
\text { Montilla (2005) } \\
\text { Sandoya (2005) } \\
\text { Del Carmen y Pedrinaci (2007) } \\
\text { Hernández (2007) } \\
\text { Godoy y Sánchez (2007) }\end{array}$ & $\begin{array}{l}\text { Ortega (2001) } \\
\text { García de la Vega (2004) } \\
\text { Parra et al. (2008) } \\
\text { Sánchez-Ferrezuelo } \\
\text { (2010) }\end{array}$ & $\begin{array}{l}\text { Piñeira y Romar (2001) } \\
\text { Bohigas y Montenegro (2000) } \\
\text { Marrón (2001) } \\
\text { Ruiz-Fernández (2002) } \\
\text { Fernández (2003) } \\
\text { Insa (2002) } \\
\text { Morales (2002) } \\
\text { Olave (2005) } \\
\text { Delgado y Ojeda (2007) } \\
\text { De los Reyes (2009) } \\
\text { Martínez y García (2008) } \\
\text { Mínguez (2010) }\end{array}$ & \\
\hline 2010-2018 & $\begin{array}{l}\text { Souto (2010; 2011) } \\
\text { Gómez-Ruíz (2010) } \\
\text { Sánchez (2010) } \\
\text { Lara (2011) } \\
\text { Gómez y Sanz (2013) } \\
\text { López y Segura (2013) } \\
\text { Samper et al. (2013) } \\
\text { García de la Vega (2014) } \\
\text { Álvarez et al. (2016) } \\
\text { Almuedo (2016) } \\
\text { García et al. (2018) } \\
\text { Sempere-Souvannavong } \\
\text { et al. (2017) }\end{array}$ & $\begin{array}{l}\text { García Martín (2014) } \\
\text { Santana et al. (2015) } \\
\text { Cuello y Cuello (2016) } \\
\text { Ensabella (2016) }\end{array}$ & $\begin{array}{l}\text { Sebastià y Blanes (2010) } \\
\text { García y García (2011) } \\
\text { Crespo (2012) } \\
\text { Delgado y Rodrigo (2012) } \\
\text { Rubio (2012) } \\
\text { Delgado et al. (2013) } \\
\text { Valenzuela (2012) } \\
\text { Santolaria (2014) } \\
\text { Martínez Fernández } \\
\text { et al. (2015) } \\
\text { Medina et al (2016) } \\
\text { Ortega et al. (2016) } \\
\text { Serrano et al. (2016) } \\
\text { Sousa et al. (2016) } \\
\text { Bullón (2017) } \\
\text { Gómez et al. (2017) } \\
\text { Fernández (2017) } \\
\text { López y Peral (2017) } \\
\text { Morote (2017) }\end{array}$ & $\begin{array}{l}\text { García y Delgado (2013) } \\
\text { Moreno et al. (2014) } \\
\text { Moreno et al. (2015) } \\
\text { Fombona y Pascual (2016) } \\
\text { Moreno (2016) } \\
\text { Rodríguez y Muñoz (2016) } \\
\text { Moreno y Vera (2017) }\end{array}$ \\
\hline
\end{tabular}

Fuente: Elaboración propia. 
Algunas de las afirmaciones y potencialidades didácticas que ofrecen algunos de los autores nombrados anteriormente, son, por ejemplo, los de Meaza (1987: 220) que considera que "a través de las salidas de campo se desarrolla en los estudiantes la sensibilidad percepcional para el análisis geográfico, propicia la observación, la investigación y la valoración científica del paisaje, lo cual permite superar las limitaciones impuestas por la cultura del asfalto". Respecto al análisis del paisaje y los conflictos territoriales y ambientales, Sánchez-Ferrezuelo (2010) argumenta que la estrategia didáctica de estos recursos posee un valor incalculable en sus actividades académicas, considerándolo insustituible e irrenunciable y, entendiéndolo como una actividad educadora que implica un contacto directo con el medio. En este sentido, según este autor, las salidas de campo se consideran como un recurso ampliamente utilizado en casi todos los ámbitos disciplinares ya que es una forma de poner en contacto al alumnado con la realidad.

La tercera temática identificada se relaciona con la propuesta de salidas de campo como estudios de caso. Por número de trabajos destaca por ser la segunda línea de estudio con un total de 31 trabajos (el 34,44\%). Sin embargo, a diferencia de lo que sucedía en las dos anteriores temáticas, en ésta, la mayor producción científica comienza a partir de la primera década del siglo XXI y convirtiéndose en los últimos años en la principal (ver Figura 2). Algunos de los trabajos de la primera década del 2000 son Bohigas y Montenegro (2000), Piñeira y Romar (2001), Marrón (2001), Insa (2002), Morales (2002), Fernández (2003), Olave (2005), Delgado y Ojeda (2007), Martínez y García (2008), De los Reyes (2009) mientras que en la última década destacan García y Ruiz (2011), Delgado y Rodrigo (2012), Crespo (2012), Rubio (2012), Valenzuela (2012), Delgado et al. (2013), Santolaria. (2014), Martínez-Fernández et al. (2015), Bullón (2017), Férnandez (2017) o Morote (2017). Cabe indicar que respecto a estas propuestas de salida de campo destacan tanto aquellas que se relacionan con el medio natural y/o rural (Fernández, 2003; Crespo, 2012; Rubio, 2012) como las que hacen referencia al análisis de espacios urbanos (Bohigas y Montenegro, 2000; Insa, 2002, De Los Reyes, 2009, Santolaria 2014; Martínez-Fernández et al. 2015). Además, se encuentran otras propuestas relacionadas con la visita a museos (Valenzuela, 2012) y la relación entre la Geografía y la Literatura como es el caso del trabajo de Marrón (2001) mediante de un itinerario didáctico para para la enseñanza-aprendizaje de la Geografía a partir del Lazarillo de Tormes.

Algunas de las potencialidades didácticas que ofrecen estas propuestas son, como explican Delgado y Rodrigo (2012), necesarias por dos motivos: 1) Para combatir los prejuicios existentes en torno a una enseñanza de la Geografía academicista y de corte muy tradicional, muy orientada a los conocimientos teóricos, fomentando el desarrollo de los conocimientos prácticos y las destrezas; y 2) Para fomentar la expansión de una cultura geográfica que también influya en un incremento del interés por parte del alumnado hacia los estudios universitarios de Geografía. Marrón (2001) ha enfatizado el indiscutible potencial y ventajas didácticas de las salidas de campo en la enseñanza de las Ciencias Sociales en general y de la Geografía en particular, coincidiendo en que la visita al territorio ofrece al alumnado una posibilidad de comprensión y relación de 
los hechos geográficos que, difícilmente serían alcanzables por medio de otros recursos. En este sentido, como ha sido enfatizado por destacados pedagogos, como María Montessori, "para enseñar y lograr aprendizajes significativos, nada mejor que el contacto directo con el entorno..." (Cordero, 2005: 95). Por ello, las salidas de campo, como indican Martínez-Fernández et al. (2015), son el método por excelencia para la enseñanza y el aprendizaje de la Geografía (observación directa sobre el terreno de las formas y procesos espaciales). Las salidas de campo, por tanto, suponen una herramienta de primer orden para el descubrimiento, comprensión e interrelación de los elementos físicos que conforman los paisajes y, como indica Crespo (2012), su realización conlleva un aprendizaje en el que esos elementos y su papel en el escenario son entendidos como una unidad funcional.

Además, Delgado y Rodrigo (2012) explican que los objetivos generales que deben perseguir la realización de las salidas de campo y, por tanto, que tienen que ser tenidas en cuenta antes de su realización, deben ser: 1) La enseñanza del vocabulario geográfico que, en contacto directo gana verdadero sentido; 2) La experiencia directa de las distancias, inclinaciones y alturas, difíciles de experimentar dentro de un aula; 3) La comparación del terreno con el mapa, extrapolando los elementos existentes y sus dimensiones del modelo a la realidad; 4) La mejor comprensión de las formas de la superficie, incluso de las formas imperfectas del paisaje, acostumbrados a los "ejemplos de libro", pero no por ello faltos de significación; 5) La comprensión de las áreas diferenciales y de la naturaleza de los límites, haciendo hincapié en los aspectos que las diferencian y la forma de transición entre unas y otras; 6) La introducción de nuevas ideas, basándose en la realidad y en la experiencia directa, lo que enriquece el proceso de aprendizaje; y 7) La interpretación de fotografías aéreas.

También, algunas de las cuestiones que se tratan en estas propuestas son las actividades a realizar durante la jornada de campo y su evaluación. Al respecto, según Marrón (2001), las formas de poder evaluar y analizar si el alumnado ha adquirido los conocimientos y ha cumplido con los objetivos propuestos puede realizarse posteriormente también en clase. Para ello, las actividades dependerán de la edad y del nivel escolar (Primaria, Secundaria, Bachillerato, Estudios Universitarios). Estas actividades pueden optimizar los resultados si se llevan a cabo trabajos que afiancen los conceptos abordados. Esta autora propone tres fases en la organización del trabajo: 1) Clasificación y análisis de la información y materiales recogidos durante la salida; 2) Interpretación de su significado y establecimiento de interconexiones; y 3) Expresión material de los resultados alcanzados. Por su parte, para la evaluación de la salida de campo, Morote (2017) (para el caso de la visita al Parque Inundable "La Marjal" de la ciudad de Alicante), explica que hay diversos recursos mediante los que se pueden sintetizar y expresar la información obtenida y la evaluación, como es el caso de la realización de cartografía. Esta actividad, obviamente, dependerá de la edad y del nivel educativo. Puede consistir en cartografiar los distintos elementos analizados y visitados (suelo urbano, zonas agrícolas abandonadas, zonas de inundación, etc.), y superponer los mapas o capas con el fin 
de descubrir las relaciones que existen entre ellos. Dicho autor explica que esta actividad desarrolla las habilidades espaciales y cartográficas y supone una de las mejores herramientas para comprender la estructura y dinámica de un paisaje. Además, éste autor propone la realización de presentaciones y murales interpretativos dedicados a elementos concretos o bien como visión del conjunto del territorio visitado. El mismo contenido puede formar parte de presentaciones a realizar en el aula. En cuarto lugar, también destaca que es interesante la realización de una memoria tomando como ejemplo las guías visuales de viaje en la que se puedan elaborar documentos en el que con todos los recursos posibles (fotografías, dibujos, siluetas, gráficos o mapas) se pueda elaborar una guía interpretativa del conjunto visitado.

Finalmente, la temática de estudio más reciente en didáctica de las salidas de campo para el caso español es la que vincula éstas con las nuevas Tecnologías de la Información y la Comunicación (el 7,78\% de los trabajos identificados). Se trata de un tema de estudio que ha surgido en los últimos diez años y que se relaciona con la potencialidad que tiene el uso de estas tecnologías en las salidas de campo de Geografía gracias al desarrollo de competencias a través de la geolocalización (García y Delgado, 2013; Fombona y Pascual, 2016) o el uso de herramientas como son los códigos QR (Moreno, 2016; Moreno et al., 2014; Moreno et al., 2015; Rodríguez y Muñoz, 2015; Moreno y Vera, 2017). Especialmente destacan los estudios sobre el uso de estos códigos en las salidas de campo, tanto en las clases de Geografía como en las de Ciencias Sociales (Educación Primaria) (Moreno et al., 2014; Moreno et al., 2015; Moreno, 2016). Estos trabajos ponen de manifiesto las potencialidades didácticas que tiene el m-learning (aprendizaje electrónico móvil) ya que es una forma de aprendizaje en el que se facilita la construcción del conocimiento, la resolución de problemas y el desarrollo de destrezas y habilidades diversas de manera autónoma, gracias al uso de dispositivos móviles portátiles (smarthphones, PDA, tabletas, Pocket PC, iPod, etc.) (Rodríguez y Muñoz, 2015).

Estas aplicaciones de uso diario permiten acceder in-situ a una gran cantidad de información así como la posibilidad de que el alumnado realice sus propios trabajos de investigación sobre el área de estudio que se visita durante la salida de campo. Por ejemplo, en el trabajo de Moreno y Vera (2017) se exponen las competencias que desarrollan los estudiantes a partir del m-learning y los códigos QR con la observación de hechos geográficos relacionados con la sociedad. Sin embargo, a pesar de que el progreso de estas tecnologías y su uso en las salidas de campo ha sido notable en la última década, actualmente su implementación aun presenta importantes problemas. En este sentido, uno de los principales inconvenientes a la hora de usar el Web-mapping y los SIG en la Didáctica de la Geografía es que los estudiantes dominan más y mejor estas tecnologías que el propio profesorado y además, éste suele tener reticencias a utilizarlo por miedo a quedar en desventaja respecto a sus estudiantes (Moreno et al., 2015; Moreno y Vera, 2017). 


\section{Conclusiones}

Con este trabajo se ha enfatizado como las salidas de campo se convierten en un recurso didáctico de primer orden para la enseñanza de la Geografía. Ello tiene que ver, debido a que ésta, se trata de una ciencia en el que intervienen fenómenos naturales y humanos, haciendo indispensable la visita al territorio para conocer de primera mano un problema o situación determinada. Más si cabe, en la actualidad, si se tiene en cuenta la importancia de la resolución de problemas de estudios de caso en el que, desde la Geografía, se acude para resolverlos. Es por ello que este recurso debería considerarse como obligatorio en las materias relacionadas con las Ciencias Sociales y Ambientales en todos sus niveles (Infantil, Primaria, Secundaria, Bachillerato, y estudios universitarios).

En relación con la identificación de las temáticas de estudio, se evidencian diferentes conclusiones. La primera es la predominancia de dos líneas principales de trabajo para el periodo analizado (1980-2018) como son los relacionados con la potencialidad que tienen las salidas de campo para la enseñanza de la Geografía y la vinculada con las ventajas que ofrece este recurso para la interpretación y análisis del territorio. Son líneas de estudio en el que se han incrementado notablemente sus publicaciones desde los años ochenta y mantienen un ritmo constante de producción hasta la actualidad. Y ello, como se ha puesto de manifiesto a lo largo de esta investigación se debe por las potencialidades didácticas y el desarrollo de competencias que se pueden adquirir: 1) El estudiante puede comprender y analizar la situación pasada, actual y futura de un territorio; 2) Desarrollar con sentido y espíritu crítico la problemática de un área determinada y dar soluciones; 3) Desarrollar la capacidad de presentar nuevas situaciones problemáticas en el área estudiada; 5) Enfrentamiento a problemas y casos reales; 6) Poner en práctica lo visto en clase; 7) Fomentar la búsqueda de información y datos para comprender la situación actual de un territorio; 8) Fomentar el trabajo cooperativo; 9) Manejo de instrumentos y técnicas de muestreo; y 10) Motivación en el aprendizaje. Son, por tanto, trabajos que siempre han tenido un peso importante en estas últimas cuatro décadas.

En segundo lugar, cabe indicar una tercera temática que, especialmente ha tenido un notable desarrollo en el s. XXI (las propuestas de salidas de campo) con el objetivo de dar a conocer y proponer ejemplos de estudios de caso. En la última década se ha convertido en la principal línea de trabajo con el 45,23\% de los estudios. En tercer lugar, se ha constatado como ha emergido en los últimos años una nueva línea de estudio vinculada con el uso de las TIC en las salidas de campo. Se trata de la línea más innovadora, con mayor proyección, pero aún con importantes inconvenientes como han constatado Moreno et al. (2014), Moreno et al. (2015) y Moreno (2016). Ello, se debe por la escasa implementación de estas tecnologías en el aula y la reticencia por parte del profesorado a usarlas, principalmente por el desconocimiento real de las potencialidades que ofrece y por no saber manejarlas. Ello indica, a día de hoy, un gran desinterés por su uso, no sólo en las clases, sino también en las salidas de campo. 
Una cuarta conclusión es que prácticamente la totalidad de los trabajos identificados han sido publicados en castellano y en editoriales españolas, lo que pone de manifiesto una escasa internacionalización de este tema de estudio. Y, a su vez, un reducido número de trabajos (teniendo en cuenta los objetivos y criterios de búsqueda de este trabajo (artículos, libros y capítulos de libro). Ello evidencia el poco peso nacional e internacional que tiene la Didáctica de la Geografía en España respecto a otras ramas (Paisaje, Geografía Rural, Turismo, Geografía Urbana, SIG, Ordenación del Territorio, Geografía de la Población, Riesgos Naturales, etc.), como ya fue puesto de manifiesto por Lasanta y Martín-Vide (2013). Ello, no sólo repercute a la propia rama didáctica sino que también, como mal mayor (entre otros factores), a la preocupación actual del reducido número de matriculados en los grados de Geografía como ha dejado constancia el último informe de la Asociación Española de Geografía (2018). Es algo que ya argumentaron De Lázaro et al. (2013): Defender que la docencia en Geografía de la E.S.O., al menos en el segundo ciclo sea impartida por geógrafos; fomentar el trabajo de campo, como laboratorio y base de la Geografía; e impulsar la innovación en la Didáctica de la Geografía, no sólo en las herramientas a emplear (TIC) sino también en las metodologías que necesariamente las deben acompañar. Por tanto, temas de interés que se han identificado en las diferentes líneas de estudio en esta investigación.

Finalmente, cabe indicar que la Didáctica de la Geografía se enfrenta a diferentes retos como la internacionalización, uso de las nuevas tecnologías como herramienta docente y la continuación de trabajos que se han venido produciendo en las últimas décadas gracias, entre otros, a la labor del Grupo de Didáctica de la Geografía. Para el caso que ocupa esta investigación (el caso de las salidas de campo), se ha podido comprobar cómo se trata de un recurso de notable interés para la enseñanza de la disciplina geográfica al acercar al alumnado una realidad que difícilmente se puede aprender sin salir del aula. Queda por ver cómo las nuevas tecnologías pueden tener una mayor implementación y uso en las salidas de campo y cómo el profesorado pueda o tenga la intención de adaptarse a éstas.

\section{Bibliografía}

Anđelkovi , S., Dedjanski, V. y Pejic, B., 2018. Pedagogical benefits of fieldwork of the students at the Faculty of Geography in the light of the Bologna Process. Journal of Geography in Higher Education, 42(1), 110-125.

Almuedo Palma, J., 2016. Los paseos escolares en las escuelas sevillanas a principios del xx: algo más que un intento de innovación didáctica. En Alanís et al. (eds.). Nativos digitales y geografía en el siglo XXI: Educación geográfica y sistemas de aprendizaje. Sevilla: Universidad Pablo de Olavide y Grupo de Didáctica de la AGE, 436-440.

Álvarez Piñeros, D., Vásquez Ortiz, W.F. y Rodríguez Pizzinato, L.A., 2016. La salida de campo, una posibilidad en la formación inicial docente. Didáctica de las Ciencias Experimentales y Sociales, 31 (2), 61-77. 
Asociación Española de Geografía. 2018. Informe de la Vocalía de Enseñanzas Universitarias. La Geografía en las titulaciones de grado y máster en las universidades de España. Asociación Española de Geografía, 132 p.

Bailey, P., 1981. Didáctica de la Geografía. Madrid: Editorial Cincel.

Bohigas, G. y Montenegro, J., 2000. Trabajos de campo e itinerarios urbanos. Un recorrido por Gràcia (Barcelona). Biblio 3W, Revista Bibliográfica de Geografía y Ciencias Sociales. Universidad de Barcelona, 222.

Boyle, A., Maguire, S., Marti, A., Milsom, C., Nash, R., Rawlinson, S., Turner, A., Wurthmann, S. y Conchie, S., 2007. Fieldwork is good: the student perception and the affective domain. Journal of Geography in Higher Education, 31, 2, 299-317.

Bullón Mata, T., 2017. La montaña junto a una gran ciudad. Inicio del excursionismo y transformación del paisaje en el valle de la Fuenfría de la Sierra de Guadarrama. Treballs de la Societat Catalana de Geografia, (Ejemplar dedicado a: Col.loqui internacional en Geohumanitats), 84, 251-264.

Clary, M., 1992. Aprender a situarse, situarse para aprender. Boletín de Didáctica de las Ciencias Sociales, 5 .

Cordero, R., 2005. María Montessori y el medio ambiente como método activo. Veinte experiencias educativas exitosas en el mundo. Nuevo León: Centro de Altos Estudios e Investigación Pedagógica, 89-100.

Cortés Samper, C., Espinosa Seguí, A., Cutillas Orgilés, E., Martínez Puche, A., Valero Escandell, J.R. y Sempere-Souvannavong, J.D., 2013. Las Salidas de Campo como recurso educativo. Valoración por parte de los alumnos y propuesta de mejora. Álvarez Teruel, J.D., Tortosa Ybáñez, Ma‥T. y Neus Pellín Buades, N. (coord.). Diseño de acciones de investigación en docencia universitaria, 2654-2667.

Crespo Castellanos, J.M., 2012. Un itinerario didáctico para la interpretación de los elementos físicos de los paisajes de la Sierra de Guadarrama. Didáctica Geográfica, 13, 15-34.

Cuello Gijón, A. y Cuello Pérez, M.I., 2016. La carretera enseña sus paisajes. Guías didácticas. Sevilla: Junta de Andalucía, Centro de Estudios Paisaje y Territorio y Universidad de Sevilla.

De Lázaro y Torres, Mํㅗ. L., Sebastiá Alcaraz, R., Tonda Monllor, E., Marrón Gaite, Mํa . J. y González Ortíz, J.L., 2013. Grupo de Didáctica de la Geografía. En Lasanta Martínez, T. y MartínVide, J. (eds.). La investigación Geográfica en España (1990-2012), 377-387.

De los Reyes, J.L., 2009. Mi casa, mi calle, mi ciudad: experiencias sobre el espacio infantil en el Madrid histórico. Terra Plural, 3, 1, 9-27.

Del Carmen, L., Pedrinaci E., 1997. El uso del entorno y el trabajo de campo. En Del Carmen, L. (Coord). La enseñanza y el aprendizaje de las Ciencias de la Naturaleza en la Educación Secundaria. Barcelona: Editorial Horsori, 133-154.

Delgado Bujalance, B. y Ojeda Rivera, J., 2007. El viaje pedagógico como método de conocimiento de paisajes. Aplicación a Andalucía. Investigaciones Geográficas, 44, 5-31.

Delgado Bujalance, B., Torres Gutiérrez, F.J., García García, A. y Ojeda Rivera, J.F., 2013. El viaje como aprendizaje significativo y pertinente. Crónica de una experiencia andaluza. En Paneque, P. y Ojeda, J.F. (eds.). El viaje en la geografía moderna. Sevilla: Universidad Internacional de Andalucía, 429-444. 
Delgado Peña, J.J. y Rodrigo Comino, J., 2012. El trabajo de campo y las competencias geográficas en el estímulo para el estudio de la Geografía: aplicación en un aula de $2^{\circ}$ de bachillerato. Didáctica Geográfica, 13, 35-56.

Delors, J., 1997. La Educación es un tesoro. Informe de la UNESCO Comisión.

De Miguel González, R., 2013a. Aprendizaje por descubrimiento, enseñanza activa y geoinformación: Hacia una didáctica de la Geografía Innovadora. Didáctica Geográfica, 14, 17-36.

De Miguel, R., 2013b. Geoinformación e innovación en la enseñanza-aprendizaje de la geografía: un reto pendiente en los libros de texto de secundaria. Didáctica de las Ciencias Experimentales y Sociales, 27, 67-90.

De Miguel González, R., 2017. La producción científica reciente en didáctica de la geografía a través de las sociedades geográficas. Declaraciones, publicaciones y proyectos a nivel nacional e internacional. Documents d'Análisi Geogràfica, vol. 63/3, 545-559.

De Miguel González, R. y Fernández de Arróyabe, P., 2016. Declaración internacional sobre educación geográfica. Didáctica Geográfica, 17, 257-274.

Ensabella, B., 2016. Trabajo de campo en geografía. Experiencia en región con conflictos socioambientales. Geograficando, Vol. 12, 1.

Fernández, J., 2003. Recursos didácticos en Geografía Física: Itinerario pedagógico sobre el paisaje natural del Oriente de Asturias. Espacio Tiempo y Forma, Serie VI, 15, 147-163.

Fernández Portela, J., 2017. La salida de campo como recurso didáctico para conocer el espacio geográfico: El caso de la ciudad de Valladolid y de Soria. Didáctica Geográfica, 18, 91-109.

Fidalgo Hijano, C., 1997. El trabajo de campo en Biogeografía. Didáctica Geográfica, 2, 33-44.

Fombona Cadavieco, J. y Pascual Sevillano, Ma. A., 2016. Investigación sobre la Tecnología de Geolocalización en las salidas de campo. En Roig-Vila, R. (coord.). Tecnología, innovación e investigación en los procesos de enseñanza-aprendizaje, 2455-2463.

France, D. y Haigh, M., 2018. Fieldwork@40: fieldwork in geography higher education. Journal of Geography in Higher Education, 42 (4), 498-514.

Friera, F., 1995. Didáctica de las Ciencias Sociales. Geografía e Historia. Madrid: Ediciones de la Torre.

Fuller, I., Edmondson, S., France, D., Higgitt, D. y Ratinen, I., 2006. International Perspectives on the Effectiveness of Geography Fieldwork for Learning.Journal of Geography in Higher Education, 30(1), 89-101.

García de la Vega, A., 2004. El itinerario geográfico como recurso didáctico para la valoración del paisaje. Didáctica Geográfica, 6, 79-95.

García Martín, M., 2014. Conflictos territoriales en torno al agua: salir al campo como metodología de enseñanza. En Martínez Medina, R. y Tonda Monllor, E.M. (eds.). Nuevas perspectivas conceptuales y metodológicas para la educación geográfica. Córdoba: Grupo de Didáctica de la Geografía de la AGE- Universidad de Córdoba, vol. 2, 179-194.

García Ruiz, A.L., 1994. Los itinerarios didácticos: una de las claves para la enseñanza y comprensión de la geografía. Iber, revista de Didáctica de las ciencias sociales, geografía e historia, 1, 117-125. 
García Ruiz, A.L., 1997. El proceso de desarrollo de los Itinerarios Geográficos. Didáctica Geográfica, 2, 1-9.

García Mestanza, J. y Delgado Peña, J.J., 2014. El desarrollo de competencias clave a través de la geolocalización. Presentación de un caso. En Ramiro Sánchez, Mํㅗ. T., Ramiro Sánchez, T. y Bermúdez Sánchez, Mํ..P. (coord.). FECIES 2013: X Foro Internacional sobre Evaluación de la Calidad de la Investigación y de la Educación Superior. 468-478.

García, S. y García, I., 2011. Itinerario didáctico por los hospitales de la Valencia en guerra. Didáctica de las Ciencias Experimentales y Sociales, 25, 165-175.

García Martín, M., Villar Lama, A., Fraile Jurado, P., Sánchez Carnero, N. y Márquez Pérez, J., 2018. Se hace geografía al andar: la salida de campo itinerante y senderista. Didáctica Geográfica, 19, 103-125.

Godoy, I. y Sánchez, A., 2007. El trabajo de campo en la enseñanza de la Geografía. Sapiens: Revista Universitaria de Investigación, 2, 137-146.

Gómez Ortiz, A., 1986. Los itinerarios pedagógicos como recurso didáctico en la enseñanza de Geografía en EGB. Didáctica Geográfica, 14, 109-116.

Gómez Ruiz, Mํㅗㄴ. 2010. El tratamiento de la información a partir de fuentes cartográficas como procedimiento esencial de la enseñanza-aprendizaje de la Geografía en el bachillerato. En: Marrón Gaite. Ma.J y De Lázaro y Torres, Ma․ (Eds.). Geografía, Educación y Formación del Profesorado en el Marco del Espacio Europeo de Educación Superior. 329-344.

Gómez Trigueros, I., 2010. Análisis del paisaje físico y humano de la provincia de Alicante: Google Earth como herramienta docente en las clases de Geografía. Geographos. Revista digital para estudiantes de Geografia y Ciencias Sociales, 1 (1), 1-26.

Gómez Gonçalves, A., Corrochano Fernández, D. y Gabriel Parra Nieto, G., 2017. Itinerarios didáctico-naturales en Educación Primaria: el noroeste de Zamora. Didáctica Geográfica, 18, $111-131$.

Gómez Mendoza, J. y Sanz Herráiz, C., 2013. La excursión geográfica universitaria con fines formativos e investigadores: su consolidación en la Escuela Española. En Paneque, P. y Ojeda, J.F. (eds.). El viaje en la geografía moderna. Sevilla: UNIA, 289-328.

Grubar, V.B., Kovačič, G., Kolega, N. y Koderman, M., 2018. Fieldwork as part of the practical part of the general matura examination in the subject of geography. Geografija v Soli, 26(2), 38-45.

Haklar, M., Singleton, A. y Parker, C., 2008. Web-mapping 2.0: the neogeography of the Geoweb. Geography Compass, 2 (6), 2011-2039.

Hernández, F.X., 2007. Didáctica de las ciencias sociales, geografía e historia. $3^{\underline{a}}$ ed. Barcelona: Graó.

Insa Sauras, Y., 2002. Itinerarios urbanos, recursos y materiales didácticos para explicar la ciudad. Iber, 32, 79-95.

Lasanta Martínez, T. y Martín-Vide, J., 2013. La investigación Geográfica en España (1990-2012). Madrid: Asociación de Geógrafos Españoles, Instituto Pirenaico de Ecología (CSIC), Centro de Ciencias Humanas y Sociales (CSIC). 510 p. 
Lara, S., 2011. Las vivencias estudiantiles del trabajo de campo y sus implicaciones pedagógicas. Revista de investigación, 35 (73), 195-218.

Liceras, A., 1997. La observación y el estudio del paisaje. En García, A.L. (coord.), Didáctica de las Ciencias Sociales, Geografía e Historia en la Enseñanza Secundaria, 297-325.

López, J.A., 2000. Las salidas de campo: mucho más que una excursión. Educar en el 2000: Educarm, 100-103.

López Fernández, J.A. y Peral Velasco, A.J., 2017. Las Vías Verdes: escenario para trabajar el medio rural en Educación Primaria. Didáctica Geográfica, 18, 171-192.

López, F. y Segura, J., 2013. Los itinerarios didácticos: un recurso interdisciplinar y vertebrador del currículum. Espiral, Cuadernos del Profesorado. Revista Digital del Centro de Profesorado Cuevas-Olula. Vol. 6, 12. [En Línea]. Disponible en: http://www.cepcuevasolula.es/espiral

Marti Hennberg, J., 1994. L'excursionisme científic i la seva contribució a les ciències naturals $i$ a la geografía, Barcelona: Alta-Fulla.

Marti Hennberg, J., 1996. El excursionismo: entre la ciencia y la estética. Mundo científico, 173, 962-969.

Martínez, L. y García, S., 2008. Itinerarios didácticos por fuente Álamo (Murcia) una estrategia educativa de innovación en el proceso de enseñanza aprendizaje en Educación Secundaria. Espiral. Cuadernos del Profesorado, (1), 1-8.

Martínez Fernández, L.C., Fernández-Vega Peláez, B. y Molina de la Torre, I., 2015. Propuesta didáctica para la interpretación del espacio geográfico: La ciudad de Segovia y su entorno. Didáctica Geográfica, 16, 135-164.

Martínez, N., Valls, R. y Pineda, F., 2009. El uso del libro de texto de Historia de España en Bachillerato: diez años de estudio (1993-2003) y dos reformas (LGE-LOGSE). Didáctica de las Ciencias Experimentales y Sociales, 23, 3-35.

Marrón Gaite, Mํa J., 2001. Geografía y Literatura. Un itinerario didáctico para la enseñanza-aprendizaje de la Geografía a partir del Lazarillo de Tormes. En Marrón Gaite, M. J. (Eds.), La formación geográfica de los ciudadanos en el cambio de milenio.

Marvell, A. y Simm, D., 2018. Emotional geographies experienced during international fieldwork: an evaluation of teaching and learning strategies for reflective assessment. Journal of Geography in Higher Education, 42(4), 515-530.

Meaza, G., 1987. La ejercitación de la sensibilidad percepcional en la didáctica de la geografía de campo. Lurralde Investigación. Espacial, 10, 219-229.

Melcón Beltrán, J., 1991. Las excursiones escolares y la educación integral. Estudios Geográficos, Vol. 52, 203, 239-262.

Mínguez, M.C., 2010. El paisaje como objeto de estudio de la Geografía. Un itinerario didáctico en el marco de la semana de la ciencia de la Comunidad de Madrid. Didáctica Geográfica, $11,37-62$.

Montilla, A.. 2005. El trabajo de campo: estrategia didáctica en la enseñanza de la Geografía. Geoenseñanza, 10 (2), 187-195. 
Morales, A., 1984. El viaje en la pedagogía de la Institución Libre de Enseñanza. Estudios turísticos, 83, 85-99.

Morales, A.J., 2002. Guía didàctica per a realitzar un itinerari ecològic pel Parc Municipal Maldonado de Riba-roja de Túria. Lauro. Quaderns d història i societat.10. IDECO \& M.I. Ayto. De Lliria. Valencia, 149-160.

Moreno, J.R., 2016. Geovicente: la enseñanza de Geografía con códigos QR. En Tortosa, M.T., Álvarez, J.D. y Pellín, N. (coords.). XIV Jornadas de investigación en docencia universitaria. Alicante: Ediciones ICE.

Moreno, N. y Cely, A., 2013. La Opacidad del Paisaje: Formas, Imágenes y Tiempos Educativos. Porto Alegre: Impresa Livre.

Moreno Vera, J.R. y María Isabel Vera-Muñoz, Ma..I., 2017. El uso del QR-learning para las salidas de campo en la enseñanza de geografía. Una experiencia didáctica. Didáctica geográfica, 51, 18, 193-209.

Moreno, J.R., García, R., y Espejo, C., 2015. El web-mapping como herramienta cartográfica en la enseñanza de la Geografía. En M.T. Tortosa, M.T., Álvarez, J.D. y Pellín, N. (coords). XIII Jornadas de Redes de Investigación en docencia universitaria. Nuevas estrategias organizativas y metodológicas en la formación universitaria para responder a la necesidad de adaptación y cambio. Alicante: Ediciones ICE.

Moreno, J.R., Vera, M.I. y López, I., 2014. Development of creative and educational thinking in arts training teachers: QR codes. Sylwan Journal, 158 (12), 185-200.

Morote Seguido, A.F., 2017. El Parque Inundable "La Marjal" de Alicante (España) como propuesta didáctica para la interpretación de los espacios de riesgo de inundación. Didáctica Geografia, 18, 211-230.

Morote Seguido, A.F. y Moltó Mantero, E., 2017. El Museo del Clima de Beniarrés (Alicante). Propuesta de un recurso didáctico para la enseñanza de la Climatología. Didáctica de las Ciencias Experimentales y Sociales, 32, 1, 109-131.

Olave, D., 2005. El itinerario didáctico: una propuesta metodológica para el análisis geo-histórico local. Geoenseñanza, 10, 197-208.

Ortega Cantero, N., 1998. El descubrimiento cultural de la Sierra de Guadarrama. Madrid y la Sierra de Guadarrama, 81-113.

Ortega, N., 2004. Educación geográfica y valoración del paisaje en la institución libre de enseñanza. Boletín de la Institución Libre de Enseñanza, 55, 39-68.

Ortega Cantero, N., 2001. Paisaje y excursiones. Francisco Giner, la Institución Libre de Enseñanza y la Sierra de Guadarrama Madrid: Caja Madrid y Raíces.

Palacios Estremera, D., 1991. Propuesta de un método para la didáctica de la geografía física a traves del trabajo de campo, la indagación. Universidad de La Laguna.

Parra Bollero, M., Domínguez Carrillo, G. y Caballero Blanco, P.J., 2008. El cuaderno de campo. Un recurso para dinamizar senderos desde la educación en valores. Agora para la educación física y el deporte, 7-8, 145-158. 
Pérez De Sánchez, A. y Rodríguez, L., 2006. La Salida De Campo: Una manera de enseñar y aprender geografía, Geoenseñanza, 2, 229-234.

Piñeira Mantiñán, Mํㅗ. J.y Romar Roel, R., 2001. Los itinerarios pedagógicos en la Educación Primaria y Secundaria: una propuesta de cambio. En Marrón Gaite, Mํ. J. (coord.). La formación geográfica de los ciudadanos en el cambio de milenio. 501-508.

Piñeiro Peleteiro, M.R., 1997. El pensamiento geográfico y el trabajo de campo en el siglo XX. Didáctica geográfica, 2, 25-32.

Rikala, J., 2015. Designing a mobile learning framework for a formal educational context. Jyväskylä: University of Jyväskylä.

Rikala, J. y Kankaanranta, M., 2013. The Use of Quick Response Codes in the Classroom. Paper presented at mLearn 2012: International Conference on Mobile and Contextual Learning 2012 Proceedings.

Rodríguez Domenech, M.A. y Muñoz Espinosa, E., 2016. La enseñanza mobile learning en Geografía: los códigos QR. En Sebastiá Alcaraz, R. y Tonda Monllor, E. (coord.). La investigación e innovación en la enseñanza de la Geografía, Alicante: Universidad de Alicante y Asociación de Geógrafos Españoles, 405-418.

Rubio, P., 2012. Itinerario didáctico por la Sierra de Albarracín. Serie Geográfica, 11, 101-116.

Ruíz Fernández, J., 2002. Recursos didácticos en Geografía Física: itinerario pedagógico sobre el paisaje natural del Oriente de Asturias. Espacio, Tiempo y Forma, Serie VI, Geografía, 15, 147-163.

Santolaria Botet, A., 2014. La ciudad como recurso didáctico. Edetania: estudios y propuestas socio-educativas, 45, 235-244.

Sánchez-Ferrezuelo, E.A., 2010. Innovación e interdisciplinariedad en los itinerarios didácticos de Ciencias Sociales. Didácticas específicas, 13, 106-112.

Sánchez Ogallar, A., 1995. El Trabajo de campo y las Excursiones. En Moreno Jiménez, A y Marrón Gaite, M. J. (Eds.), Enseñar Geografía, de la teoría a la práctica. Madrid. Editorial Síntesis, 160-184.

Sánchez, M. y Godoy, I., 2002. El trabajo de campo como estrategia metodológica para la enseñanza de las Ciencias de la Tierra. Boletín Multidisciplinario, 12 (1), Fundación CENAMEC. Caracas.

Sandoya Hernández, M.A., 2005. Estrategias didácticas para jóvenes profesores de Geografía. Didáctica Geográfica, (7), 535-549.

Santana Martín, D., Morales Hernández, A.J., Colomer Rubio, J.C., Campo Pais, B. y Caurín Alonso, C., 2015. Parques Naturales: La necesaria conceptualización transformadora en la Educación Primaria y Secundaria. Didáctica Geográfica, (16), 73-94.

Sebastiá, R. y Blanes, G., 2010. El itinerario didáctico industrial: el problema de la puesta en práctica de los principios teóricos en el "Molinar" de Alcoy (Alicante). Didáctica Geográfica, 11, 111-140.

Sebastiá Alcaraz, R. y Tonda Monllor, E.M., 2011. Características y evolución de la Revista Didáctica Geográfica. Didáctica Geográfica, (12), 19-48. 
Sempere-Souvannavong, J.D., Cutillas Orgilés, E., Ana Espinosa Seguí, A., González de Bustos, L. José Ramón Valero Escandell, J.R., 2017. El trabajo de campo en Geografía Humana. En Antolí Martínez, J.M., Lledó Carreres, A., Pellín Buades, N. y Rosabel Roig-Vila, R. (coord.). Memorias del Programa de Redes-I3CE de calidad, innovación e investigación en docencia universitaria: Convocatoria 2016-17. 311-315.

Souto, X.M., 1998. Didáctica de la Geografía. Problemas sociales y conocimiento del medio. Barcelona: Ediciones del Ser.

Souto, X.M., 2010. ¿Qué escuelas de Geografía para educar en ciudadanía? Didáctica de las Ciencias Experimentales y Sociales, 24, 25-44.

Souto, X.M., 2011. "Los recursos y las estrategias didácticas para la enseñanza de la Geografía". En: J. Prats (coord.). Didáctica de la Geografía y la Historia. Barcelona: Ed. Graó, p. 159-172.

Sousa Fernandez, S.A. García, D. y Souto, X.M., 2016. Educación Geográfica y las salidas de campo como estrategia didáctica: un estudio comparativo desde el Geoforo Iberoamericano Biblio $3 W$. Revista Bibliográfica de Geografía y Ciencias Sociales. [En línea]. Barcelona: Universidad de Barcelona, 5 de abril de 2016, Vol. XXI, no 1.155. <http://www.ub.es/geocrit/b3w-1155.pdf>.

Tatjer Mir, M., 1995. El aprendizaje integrado de las Ciencias Sociales a través del medio urbano. En Boïls Tuzín, E. (coord.). El ocio en espacios urbanizados. Orientación teórica y praxis didáctica, Valencia: Nau Llibres, 17-26.

Tilling, S., 2018. Ecological science fieldwork and secondary school biology in England: does a more secure future lie in Geography?. Curriculum Journal, 29(4), 538-556.

Tucker, F. y Horton, J., 2019. The show must go on!" Fieldwork, mental health and wellbeing in Geography, Earth and Environmental Sciences. Area, Vol. 51 (1), 84-93.

Umaña De Gauthier, G., 2004. "Importancia de las salidas de campo en la enseñanza de la Geografía". Folios, 20, p. 105-120.

Valenzuela Valdivieso, E., 2012. La relación museo-escuela: verdad o utopía. Una propuesta para la asignatura de Geografía. Investigación Universitaria Multidisciplinaria: Revista de Investigación de la Universidad Simón Bolivar, 11, 7-16.

Vilarrasa Cunillé, A., 1999. Les sortides escolars: Viatge cap al coneixement. Guix: Elements d'acció educativa, 251, 41.

Vilarrasa Cunillé, A., 2003a. Salir del aula. Iber: Didáctica de las Ciencias Sociales. Geografía e Historia, 36, 5-6.

Vilarrasa, A., 2003b. Salir del aula. Reapropiarse del contexto, Íber. Didáctica de las ciencias sociales, geografía e historia, 36, 13-25.

Wass, S., 1992. Salidas escolares y trabajo de campo en la Educación Primaria. Madrid: Ministerio de Educación y Ciencia.

Zusman, P., 2011. La tradición del trabajo de campo en Geografía. Geograficando, 7 (7), 5-32. 
